\title{
Nota de egreso en el expediente clínico electrónico del Instituto Nacional de Neurología y Neurocirugía
}

\section{Discharge note in the electronic clinical record of the National Institute of Neurology and Neurosurgery}

\author{
Karen M. Castro-Gutiérrez ${ }^{1 *}$, Viviana V. Mejía-Gutiérrez ${ }^{1}$, Ma. Antonieta Ramírez-Crescencio", \\ Roberto Galán-Galán² y Adolfo Leyva-Rendón ${ }^{3}$ \\ ${ }^{1}$ Coordinación de Calidad; ${ }^{2}$ Subdirección de Servicios Auxiliares de Diagnóstico y Tratamiento; ${ }^{3}$ Dirección Médica. Instituto Nacional de Neurología y Neurocirugía \\ Manuel Velasco Suárez, Ciudad de México, México
}

\begin{abstract}
Background: The National Institute of Neurology and Neurosurgery adopted the electronic medical record with the aim of improving the availability of records, streamlining work flow and reducing errors in information. Objective: To evaluate the compliance in filling out the hospital discharge notes of patients discharged for a year and to implement actions to improve the records with reliable information. Methodology: Retrospective analysis of the discharge notes generated in the electronic medical record. The information recorded in each of the sections of the notes was evaluated. Result: In the three measurements of the audit, there was less compliance in the procedural sections (83.30 to $79.93 \%$ ) and physician data (56.05 to $75.83 \%$ ); in addition, the filling of patient data decreased. Conclusions: The implementation of the electronic medical record has been a challenge, mainly due to the lack of commitment, ignorance of professional responsibility and legal importance, confusion of classifications and resistance to the use of information technologies.
\end{abstract}

Key words: Electronic health record. Discharge summary. Information technology.

\section{RESUMEN}

Antecedentes: El Instituto Nacional de Neurología y Neurocirugía adoptó el expediente clínico electrónico con el objetivo de mejorar la disponibilidad de los registros, agilizar el flujo de trabajo y reducir los errores en la información. Objetivo: Evaluar el cumplimiento en el llenado de las notas de egreso hospitalario de pacientes dados de alta durante un año e implementar acciones que permitan mejorar los registros con información confiable. Metodología: Análisis retrospectivo de las notas de egreso generadas en el expediente clínico electrónico. Se evaluó la información registrada en cada uno de los apartados de las notas. Resultado: En las tres mediciones de la auditoría existió un menor cumplimiento en los apartados de procedimientos (83.30 a 79.93\%) y datos de los médicos (56.05 a 75.83\%); además, disminuyó el llenado de los datos del paciente. Conclusiones: La implementación del expediente clínico electrónico ha sido un reto, principalmente por la falta de compromiso, desconocimiento de la responsabilidad profesional y la importancia legal, confusión de clasificaciones y resistencia al uso de tecnologías de la información.

Palabras clave: Expediente clínico electrónico. Nota de egreso. Tecnologías de la información.

\section{Correspondence:}

*Karen M. Castro-Gutiérrez

E-mail: karenmelissa.cst@gmail.com
Date of reception: 04-02-2020

Date of acceptance: 29-09-2020

DOI: 10.24875/HMCM.20000003
Available online: 18-11-2020

Hosp Med Clin Manag. 2020;13:96-102

2604-0018 / (c) 2020 Mexican Regional Hospitals of High Specialty and Federal Hospitals. Published by Permanyer. This is an open access article under the CC BY-NC-ND license (http://creativecommons.org/licenses/by-nc-nd/4.0/). 


\section{INTRODUCCIÓN}

El 1 de septiembre de 2016 se implementó el expediente clínico electrónico (ECE) en hospitalización del Instituto Nacional de Neurología y Neurocirugía (INNN), lo que representó una oportunidad de estandarizar el diseño de los formularios utilizados durante la atención médica, así como dar cumplimiento a la NOM-004SSA3-2012 del expediente clínico.

En octubre de 2016 la Dirección General de Información en Salud (DGIS) emitió un comunicado informando que las Instituciones de Salud debían adoptar el formulario de la Nota de Egreso al Sistema Nacional de Información Básica en Materia de Salud (SINBA), por lo que se integró un equipo multidisciplinario institucional que incluyó a las áreas clínicas involucradas en el llenado de los diferentes apartados de la información requerida por la DGIS y se diseñó el formulario de la nota de egreso hospitalario (DM/NEH-1/2017) que se empezó a utilizar a partir del 1 de junio de $2017^{2}$.

Su implementación incluyó la capacitación a personal administrativo y médico, así como controles de cambio de acuerdo con las observaciones del Departamento de Epidemiología con el apoyo del Departamento de Tecnologías de Información (TIC).

En el INNN, el Departamento de Archivo Clínico otorga un folio y verifica los días de estancia hospitalaria. El Departamento de Epidemiología revisa y valida la información clínica registrada en las hojas de egreso y el Departamento de TIC envía la información a la DGIS en formato TXT. Cuando el Departamento de Epidemiología identifica errores $u$ omisiones (incidencias) en las notas de egreso, las notifica en forma periódica a la Coordinación de Calidad del INNN, con la finalidad de realizar una revisión y presentarlas en el Comité del Expediente Clínico para llevar a cabo un análisis y emitir recomendaciones.

La presente auditoría fue diseñada para identificar las incidencias ocurridas en el llenado del formulario de nota de egreso hospitalario, así como para identificar a los involucrados y de esta manera poder presentar los resultados al Comité del Expediente Clínico e implementar mejoras.

\section{CONSIDERACIONES ÉTICAS}

En el estudio no se infringieron los derechos, privacidad ni confidencialidad del paciente. No implicó compensaciones entre el costo, la calidad y la seguridad para el paciente. No supuso ningún riesgo o carga para el paciente más allá de la atención habitual. No se obtuvo información del paciente fuera de la habitual. No implicó el uso de intervenciones clínicas o sistemas no probados.

\section{OBJETIVOS}

- Implementar la nota de egreso hospitalario SINBA en el ECE del INNN.

- Evaluar el cumplimiento en el llenado de la información en el formulario de nota de egreso hospitalario en todos los pacientes que fueron dados de alta en el INNN durante el periodo señalado.

- Identificar áreas de oportunidad e implementar acciones que permitan mejorar los registros con información confiable.

\section{MÉTODOS}

Se incluyeron todos los pacientes que fueron egresados en el INNN desde el 1 de agosto de 2018 hasta el 31 de agosto de 2019, dividido en tres periodos. Se realizó una recolección de datos retrospectiva de las notas de egreso registradas en el ECE durante dicho periodo.

Para la evaluación se dividió la nota de egreso hospitalario (Fig. 1) en siete apartados: 1) datos del paciente (ficha de identificación, afiliación a los servicios de salud, habla de alguna lengua indígena, domicilio y datos del responsable legal); 2) información de la estancia hospitalaria (fecha de ingreso y de egreso, días de estancia, motivo de ingreso y servicio tratante); 3) diagnósticos finales al egreso (afección principal y comorbilidades); 4) procedimientos diagnósticos, terapéuticos y quirúrgicos (procedimientos codificados por la Clasificación Internacional de Enfermedades [CIE] 9); 5) antecedentes ginecoobstétricos (número de embarazos, abortos, partos, cesáreas y si actualmente es una mujer en edad fértil); 6) Resumen clínico (resumen de la evolución durante la estancia hospitalaria, resultados de laboratorio y gabinete, tratamientos empleados, pronóstico, recomendaciones y plan de egreso), y 7) datos de los médicos tratantes (firmas, cédulas profesionales y clave única de registro de población [CURP]). 

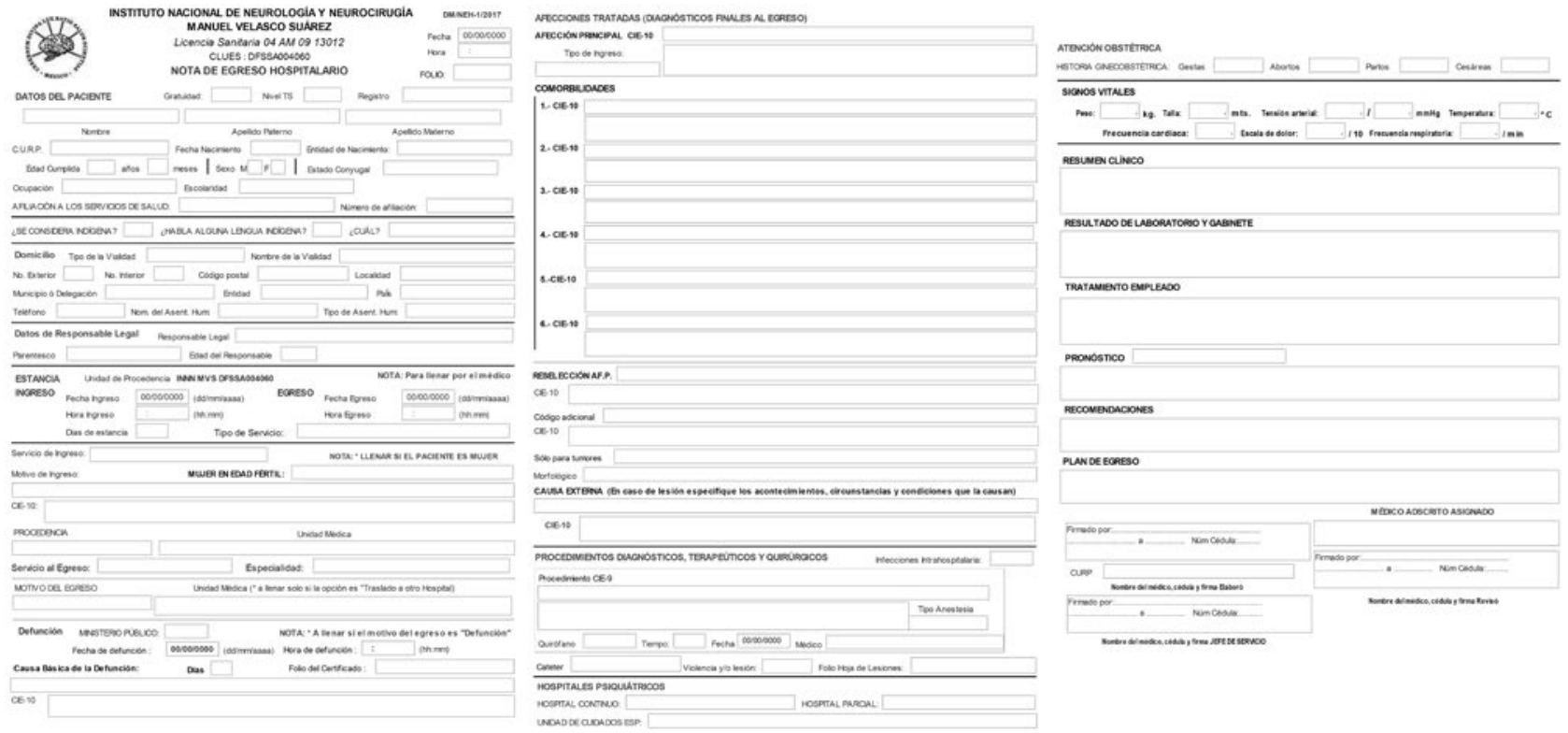

Figura 1. Nota de egreso hospitalario del Instituto Nacional de Neurología y Neurocirugía.

Los criterios para las mediciones se determinaron de acuerdo con los apartados mostrados en la tabla 1, estableciendo un cumplimiento esperado del $100 \%$ en todos los criterios, sin excepciones, ya que idealmente todos los campos deben ser llenados de forma adecuada.

La revisión se realizó de forma mensual y para el análisis de información se dividió en tres periodos. Los resultados se presentaron en las reuniones del Comité del Expediente Clínico.

\section{RESULTADOS}

\section{Primer análisis y acciones de mejora}

El primer periodo se estableció del 1 de agosto al 30 de noviembre de 2018, en el cual se dieron de alta a 927 pacientes. Se encontraron omisiones en 351 (37.86\%) notas de egreso (Fig. 2).

Los apartados con mayor cumplimiento fueron: diagnósticos finales al egreso (100\%), antecedentes ginecoobstétricos $(94.89 \%)$, resumen clínico $(89.95 \%)$, datos del paciente $(88.07 \%)$ e información de la estancia hospitalaria (87.73\%). Los apartados con menor cumplimiento fueron los procedimientos (83.30\%) (Fig. 3) y datos de los médicos (56.05\%) (Fig. 4).
Se presentaron los resultados en el Comité del Expediente Clínico y se implementaron las siguientes acciones: el director médico informó sobre los resultados a las subdirecciones y señaló la importancia del llenado de la firma electrónica por parte de médicos adscritos y jefes de servicio. Se modificó el formato para incluir la cédula profesional en la firma electrónica de todos los médicos y se aplicó un invisible en el impreso de su CURP. El apartado de «mujer en edad fértil» se estableció como un campo obligatorio. El Departamento de TIC asignó personal para el envío y registro de egresos hospitalarios a la coordinadora del sector. Se dio capacitación al personal que utiliza la nota de egreso, se elaboró un manual de apoyo para el llenado del formato y se creó una guía rápida para gestionar el alta por defunción.

\section{Segundo análisis y acciones de mejora}

El segundo periodo de análisis se realizó del 1 de diciembre de 2018 al 31 de marzo de 2019. Fueron dados de alta 898 pacientes y se reportaron omisiones en 236 hojas de egreso (26.28\%).

Los apartados con mayor cumplimiento fueron el resumen clínico $(98.71 \%)$, los diagnósticos al egreso (97.63\%), datos del paciente $(92.46 \%)$ y antecedentes ginecoobstétricos (89.87\%). 
Tabla 1. Criterios de evaluación en las notas de egreso del Sistema Nacional de Información Básica en Materia de Salud (SINBA)

\begin{tabular}{lcc}
\hline Criterio medido & $\begin{array}{c}\text { Estándar } \\
\text { (\% cumplimiento esperado) }\end{array}$ & Excepción \\
\hline Datos del paciente & 100 & Ninguna \\
Información de la estancia hospitalaria & 100 & Ninguna \\
Diagnósticos finales al egreso & 100 & Ninguna \\
Procedimientos diagnósticos, terapéuticos y quirúrgicos & 100 & Ninguna \\
Antecedentes ginecoobstétricos & 100 & Ninguna \\
Resumen clínico & 100 & Ninguna \\
Datos de los médicos tratantes & 100 & Ninguna \\
\hline
\end{tabular}

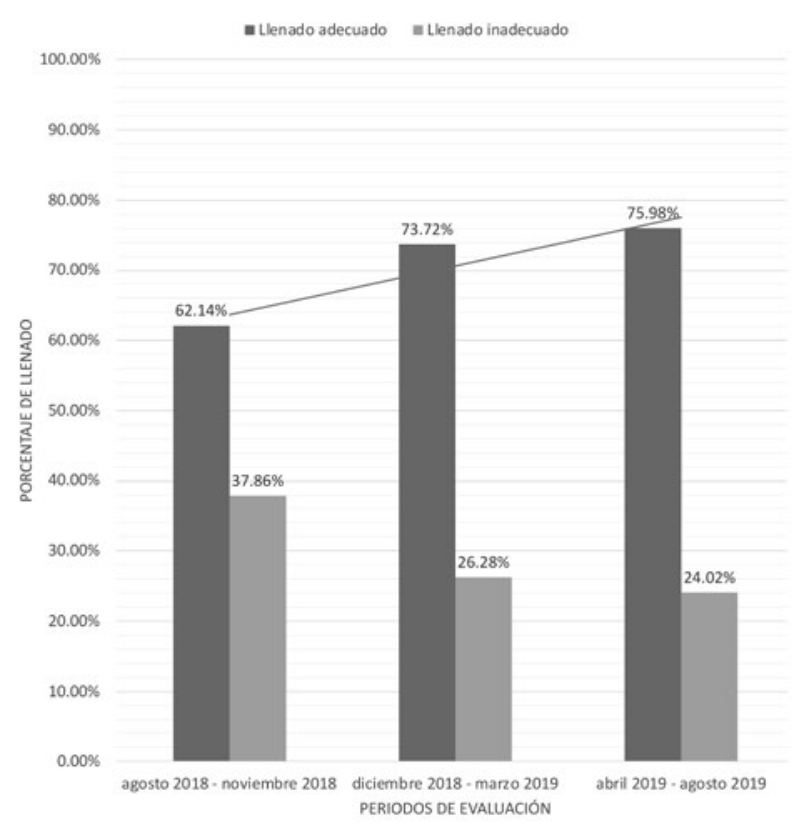

Figura 2. Porcentaje de llenado adecuado e inadecuado de los datos registrados en las notas de egreso del Instituto Nacional de Neurología y Neurocirugía (1 de agosto de 2018 - 31 de agosto de 2019).

Los apartados con menor cumplimiento fueron la información de la estancia hospitalaria (86.42\%), datos de los médicos (67.67\%) y los procedimientos (67.24\%).

Después de presentar los resultados en el Comité del Expediente Clínico se hizo una notificación mensual a los jefes de servicio sobre las omisiones ocurridas en las notas de egreso para solicitar la corrección de las incidencias. Se identificó que los médicos registraban los códigos CIE-9 y CIE-10 de forma indistinta en los apartados de diagnóstico y procedimiento, por lo que se solicitó que el diagnóstico se colocara como texto libre y el Departamento de Epidemiología fuera el encargado de codificar la información. En febrero de 2019 se capacitó a los residentes de nuevo ingreso en el uso de todos los formularios del ECE y en marzo se capacitó a los

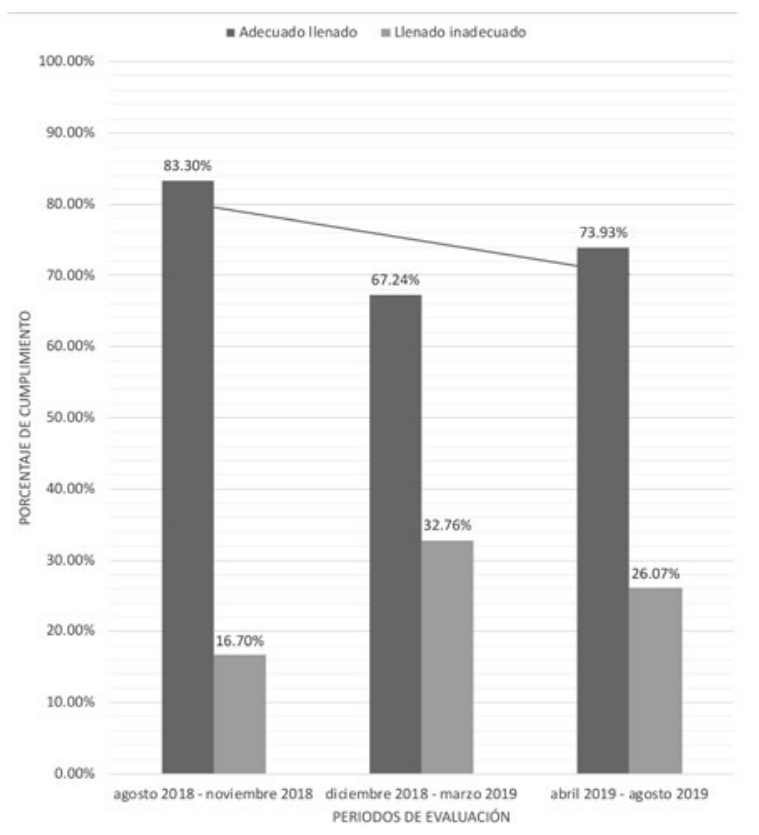

Figura 3. Porcentaje de llenado adecuado e inadecuado de procedimientos diagnósticos, terapéuticos o quirúrgicos (Clasificación Internacional de Enfermedades 9) en las notas de egreso.

residentes de primer y segundo años de las especialidades y posgrados en el llenado de la hoja de egreso.

\section{Tercer análisis y acciones de mejora}

El análisis se llevó a cabo del 1 de abril al 31 de agosto de 2019. Se registraron 1,099 altas y se encontraron omisiones en 264 hojas de egreso (24.02\%).

Los apartados con mayor cumplimiento fueron el resumen clínico (97.87\%), información de la estancia hospitalaria (96.21\%), antecedentes ginecoobstétricos (91.23\%) y diagnóstico al egreso (82.94\%). El menor cumplimiento se encontró en los datos del paciente (81.99\%), procedimientos (73.93\%) y datos de los médicos $(75.83 \%)$. 


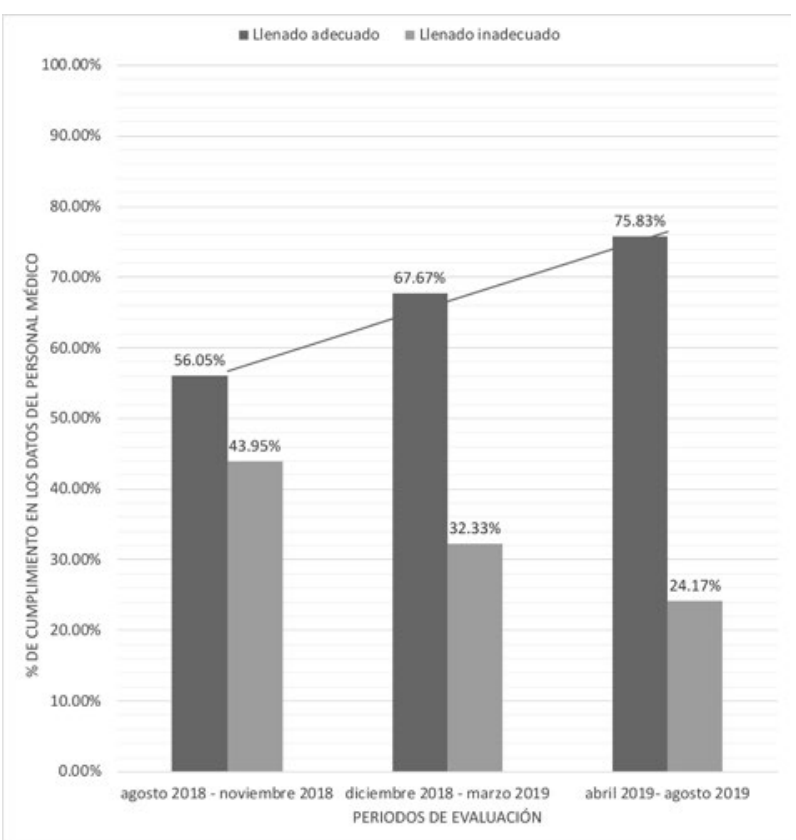

Figura 4. Porcentaje de llenado de los datos y firmas de los médicos que elaboran y revisan las notas de egreso del Instituto Nacional de Neurología y Neurocirugía.

Los resultados fueron presentados en el Comité del Expediente Clínico. Nuevamente se realizó la notificación mensual por servicios de las incidencias ocurridas en las notas de egreso y se solicitaron estrategias y/o sugerencias a la Dirección de Enseñanza para atender las irregularidades en el llenado de estas notas. El departamento de TIC capacitó a personal de nuevo ingreso en la coordinación de calidad para fungir como usuario clave e interactuar con el personal de salud que cometiera incidencias, con la finalidad de capacitarlos.

\section{DISCUSIÓN}

La nota de egreso hospitalario tiene importancia e impacto en el seguimiento de los pacientes, ya que permite comunicar a otros médicos y al propio paciente los eventos ocurridos durante su estancia hospitalaria y las recomendaciones que seguir. Algunos autores han mostrado que la falta de información en las notas de egreso puede incrementar el riesgo de eventos adversos, errores médicos y readmisión hospitalaria ${ }^{3,4}$.

El INNN adoptó el ECE a partir de abril de 2016 con el objetivo de mejorar la disponibilidad de los registros, agilizar el flujo de trabajo y reducir los errores 5 . La nota de egreso hospitalario SINBA es un ejemplo de reingeniería de los formularios utilizados durante la atención clínica hospitalaria para cumplir con la normativa establecida.

Un estudio realizado en 2003 por Rodríguez Velasco, et al., en el Instituto Mexicano del Seguro Social, proponía implementar un formulario electrónico y unificar el formato de egreso entre todas las instituciones para mejorar el llenado y la calidad de la información registrada ${ }^{6}$. Sin embargo, el INNN y otros centros hospitalarios han coincidido en que esta tarea resulta sumamente compleja.

Por medio de esta auditoría se han detectado algunas dificultades para su utilización, como la resistencia al uso de las tecnologías de la información, desconocimiento de la responsabilidad profesional del personal de salud, falta de compromiso profesional y desconocimiento de los sistemas internacionales de codificación de enfermedades y de procedimientos. Esto también fue evidenciado por Valdez Méndez, quien analizó el impacto en la calidad de los expedientes en un hospital privado antes y después de la implementación del ECE, y observó que, aunque existe una alta proporción de expedientes con resumen clínico de egreso, hay un incremento de formatos sin llenar o ausentes, condición derivada de la omisión en su elaboración por parte de los usuarios ${ }^{7}$.

Durante el análisis de la información se observó que el principal problema se encuentra en el registro de los datos y firma de los médicos tratantes. De acuerdo con las Normas Oficiales Mexicanas referentes al expediente clínico, cada prestador de servicios de salud debe ser capaz de reportar datos mínimos obligatorios para su identificación (CURP, nombre y apellidos). Además, todas las notas deben contener la firma autógrafa, electrónica o digital, según sea el caso ${ }^{8,9}$. La ausencia de cualquiera de estos datos muestra la falta de revisión y supervisión de la información, así como el incumplimiento de un documento considerado como instrumento jurídico ${ }^{10,11}$. En ese caso una buena estrategia fue modificar el formato para incluir la cédula profesional en la firma electrónica y el invisible en el impreso del CURP. Aunque no se logró un cumplimiento total, sí se logró evidenciar una mejora considerable al pasar del 56.05 al $75.83 \%$.

El llenado de procedimientos disminuyó hasta un 73.93\% de cumplimiento en la última medición. Otros autores también han coincidido en que una parte de la 
información puede ser subcodificada y la exactitud puede variar de acuerdo con el tipo de procedimiento. Los de tipo quirúrgico son mayormente reportados, mientras que los procedimientos menores se omiten con más frecuencia, en especial aquellos realizados en departamentos de radiología ${ }^{12}$. Sabemos que la codificación adecuada de los diagnósticos es esencial para tener informes estadísticos confiables que favorezcan la investigación y la docencia ${ }^{13}$. Sin embargo, nuestros resultados muestran una reducción del cumplimiento de este apartado. Se ha señalado que los problemas más frecuentes al registrar los diagnósticos son el uso de terminología diferente a las descripciones de los códigos CIE-10, errores ortográficos, estructura sintáctica incorrecta, uso de jerga médica, sinónimos, acrónimos y abreviaturas ${ }^{14,15}$

El llenado del apartado de datos personales también ha disminuido, reflejando indiferencia por parte del personal administrativo que recaba esta información, considerando que es importante establecer estrategias que mejoren la recopilación o actualización de estos datos.

Aunque existe cada vez mayor disponibilidad a la implementación de herramientas informáticas aplicadas a los registros clínicos, la resistencia de los usuarios ante estos recursos continúa generando expedientes incompletos y con deficiencias.

Después de llevar a cabo múltiples acciones, en general se observó una importante mejora en el cumplimiento de la información registrada (del 62.14 al $75.98 \%$ ); sin embargo, será necesario dirigir esfuerzos a la sensibilización del personal de salud para la adecuada utilización de estas herramientas, y se requieren estrategias para que asuma su responsabilidad y comprenda la importancia del Ilenado claro, correcto y completo de los formularios del expediente clínico, que son una evidencia del trabajo multidisciplinario.

\section{CONCLUSIÓN}

La implementación del ECE y la nota de egreso ha sido un reto. Principalmente ha influido la falta de compromiso por el personal de salud, el desconocimiento de la responsabilidad profesional e importancia legal del expediente, el desconocimiento y confusión de clasificaciones, así como la resistencia al uso de tecnologías de la información.

Durante la realización de la auditoría obtuvimos resultados variables y no todos los casos fueron satisfactorios. De los siete apartados evaluados en la nota de egreso, tres presentaron un mayor cumplimiento (información de la estancia intrahospitalaria, resumen clínico y datos de los médicos), un apartado mantuvo resultados similares en las tres mediciones (antecedentes ginecoobstétricos) y en tres apartados disminuyó gradualmente el cumplimiento (datos del paciente, diagnósticos al egreso y procedimientos). A pesar de esto pudimos observar un mayor cumplimiento general en el llenado de las notas.

De acuerdo con la experiencia obtenida, seguiremos implementando estrategias y acciones de mejora que permitan obtener información confiable, sistemática y fácil de codificar. Es imprescindible crear conciencia entre todo el personal sobre su relevancia como instrumento médico, administrativo, legal, educativo y de investigación, así como la importancia de hacer buen uso del expediente y llevar a cabo un adecuado registro de la información.

\section{FINANCIAMIENTO}

La presente investigación no ha recibido ninguna beca específica de agencias de los sectores público, comercial o sin ánimo de lucro.

\section{CONFLICTO DE INTERESES}

Los autores declaran no tener conflicto de intereses.

\section{RESPONSABILIDADES ÉTICAS}

Protección de personas y animales. Los autores declaran que para esta investigación no se han realizado experimentos en seres humanos ni en animales.

Confidencialidad de los datos. Los autores declaran que en este artículo no aparecen datos de pacientes.

Derecho a la privacidad y consentimiento informado. Los autores declaran que en este artículo no aparecen datos de pacientes. 


\section{BIBLIOGRAFÍA}

1. NORMA Oficial Mexicana NOM-004-SSA3-2012, Del Expediente Clínico [Internet]. Gobierno de México, Secretaría de Salud, Diario Oficial de la Federación; 2012. Disponible en: http://dof.gob.mx/nota_detalle_popup.php?codigo=5272787

2. Guía y formatos para el intercambio de información en salud referente al reporte de información de egresos hospitalarios [Internet]. Secretaría de Integración y Desarrollo del Sector Salud, Dirección General de Información en Salud; 2017 [consultado el 24 de enero de 2020]. Disponible en: http:// gobi.salud.gob.mx/guias/GIIS-B002-03-09-EgresosHospitalarios.pdf

3. Bergkvist A, Midlöv P, Höglund P, Larsson L, Bondesson A, Eriksson T. Improved quality in the hospital discharge summary reduces medication errors-LIMM: Landskrona Integrated Medicines Management. Eur J Clin Pharmacol. 2009;65:1037-46.

4. Talwalkar JS, Ouellette JR, Alston S, Buller GK, Cottrell D, Genese T, et al. A structured workshop to improve the quality of resident discharge summaries. J Grad Med Educ. 2012:8-91.

5. Esper GJ, Drogan 0, Henderson WS, Becker A, Avitzur 0, Hier DB. Health information technology and electronic health records in neurologic practice. Neurol Clin. 2010;28:411-27.

6. Rodríguez A, Furuya ME, Rodríguez MD, Cárdenas R, Madrigal 0, Rico MS, et al. La hoja de egreso hospitalario. Comentarios de un comité de mortalidad. Rev Med IMSS. 2003;41:481-5.

7. Valdez D. Herramientas de seguridad para el paciente: Experiencia de la implementación del expediente clínico electrónico. Cir Gen. 2011;33:146-50.

8. NORMA Oficial Mexicana NOM-024-SSA3-2010, Que establece los objetivos funcionales y funcionalidades que deberán observar los productos de Sistemas de Expediente Clínico Electrónico para garantizar la interoperabilidad, procesamiento, interpretación, confidencialidad, seguridad y uso de estándares y catálogos de la información de los registros electrónicos en salud [Internet]. México: Diario Oficial de la Federación; 30/11/212. Disponible en: http://www.dof.gob.mx/normas0ficiales/4151/salud/salud.htm\#: : text=expide $\% 201$ a $\% 20$ siguiente $\% 3 \mathrm{~A}-$, Norma $\% 200 \mathrm{ficial} \% 20 \mathrm{Mexicana} \% 20$ NOM $\% 2$ D024\%2DSSA3\%2D2010\%2C\%20Que,est $\%$ C3\%A1ndares $\% 20$ y $\% 20$ cat $\%$ C3\%A1logos $\% 20$ de $\% 201$ a

9. NORMA Oficial Mexicana NOM-024-SSA3-2012, Sistemas de información de registro electrónico para la salud. Intercambio de información en salud [Internet]. México: Diario Oficial de la Federación; 30/11/212. Disponible en: http://dof.gob.mx/nota_detalle.php?codigo=5280847\&fecha=30/11/201 2\#: :text=1.1\%20Esta\%20Norma\%20tiene\%20por,registren\%2C\%20intercambien $\% 20 y \% 20$ consoliden $\% 20$ informaci $\%$ C3\%B3n

10. Kurczyn P. Contenido e importancia del expediente clínico. Acceso y confidencialidad. Rev Fac Derecho Méx. 2019;69:893-918.

11. Lupa S, Yacaman R, Martínez E, Ruelas V. La relevancia del expediente clínico para el quehacer médico. An Med (Mex). 2015;60:237-40.

12. Quan H, Parsons GA, Ghali WA. Validity of procedure codes in International Classification of Diseases, 9th revision, clinical modification administrative data. Med Care. 2004:42:801-9.

13. Ramos AJ, Vázquez JL, Herrera S. CIE-10 (I): Introducción, historia y estructura general. Pap Méd. 2002;11:24-35.

14. Almagro M, Martínez R, Fresno V, Montalvo S. Estudio preliminar de la anotación automática de códigos CIE-10 en informes de alta hospitalarios. SEPLN. 2018;60:45-52.

15. Farzandipour M, Sheikhtaheri A, Sadoughi F. Effective factors on accuracy of principal diagnosis coding based on International Classification of Diseases, the 10th revision (ICD-10). Int J Inf Manage. 2010;30:78-84. 